\title{
Real-time measurement of the vaginal pressure profile using an optical-fiber- based instrumented speculum
}

Luke A. Parkinson

Caroline E. Gargett

Natharnia Young

Anna Rosamilia

Aditya V. Vashi

Jerome A. Werkmeister

Anthony W. Papageorgiou

John W. Arkwright 


\title{
Real-time measurement of the vaginal pressure profile using an optical-fiber-based instrumented speculum
}

\author{
Luke A. Parkinson, ${ }^{\text {a,* }}$ Caroline E. Gargett, ${ }^{\mathrm{b}, \mathrm{c}}$ Natharnia Young, ${ }^{\mathrm{b}}$ Anna Rosamilia, ${ }^{\mathrm{b}, \mathrm{c}}$ Aditya V. Vashi, ${ }^{\mathrm{d}}$ \\ Jerome A. Werkmeister, ${ }^{\mathrm{c}, \mathrm{d}}$ Anthony W. Papageorgiou, ${ }^{\mathrm{a}}$ and John W. Arkwright ${ }^{\mathrm{a}}$ \\ ${ }^{a}$ Flinders University, Medical Device Research Institute, 1284 South Road, Tonsley, SA 5042, Australia \\ ${ }^{b} H u d s o n$ Institute of Medical Research, The Ritchie Centre, 27-31 Wright Street, Clayton, VIC 3168, Australia \\ 'Monash University, Department of Obstetrics and Gynaecology, 246 Clayton Road, Clayton, VIC 3168, Australia \\ ${ }^{\mathrm{d} C S I R O}$ Manufacturing, Research Way, Clayton, VIC 3168, Australia
}

\begin{abstract}
Pelvic organ prolapse (POP) occurs when changes to the pelvic organ support structures cause descent or herniation of the pelvic organs into the vagina. Clinical evaluation of POP is a series of manual measurements known as the pelvic organ prolapse quantification (POP-Q) score. However, it fails to identify the mechanism causing POP and relies on the skills of the practitioner. We report on a modified vaginal speculum incorporating a double-helix fiber-Bragg grating structure for distributed pressure measurements along the length of the vagina and include preliminary data in an ovine model of prolapse. Vaginal pressure profiles were recorded at $10 \mathrm{~Hz}$ as the speculum was dilated incrementally up to $20 \mathrm{~mm}$. At $10-\mathrm{mm}$ dilation, nulliparous sheep showed higher mean pressures $(102 \pm 46 \mathrm{mmHg})$ than parous sheep $(39 \pm 23 \mathrm{mmHg})(P=0.02)$, attributable largely to the proximal (cervical) end of the vagina. In addition to overall pressure variations, we observed a difference in the distribution of pressure that related to POP-Q measurements adapted for the ovine anatomy, showing increased tissue laxity in the upper anterior vagina for parous ewes. We demonstrate the utility of the fiber-optic instrumented speculum for rapid distributed measurement of vaginal support. ๑ 2016 Society of Photo-Optical Instrumentation Engineers (SPIE) [DOI: 10.1117/1.JBO.21.12.127008]
\end{abstract}

Keywords: fiber-optic sensors; prolapsed; pelvic support; fiber-Bragg gratings; vaginal pressure profile.

Paper 160549R received Aug. 11, 2016; accepted for publication Nov. 29, 2016; published online Dec. $23,2016$.

\section{Introduction}

The three main contributors to the support for the pelvic organs, bladder, bowel, and uterus include the suspensory ligaments of the uterus at the cervical level, the endopelvic fascia of the vaginal wall, and the levator ani skeletal muscles of the pelvic floor. ${ }^{1,2}$ Childbirth injuries damage these support structures, which overstretch or tear, resulting in weakening of the vaginal wall and allowing descent or herniation of the pelvic organs into the vagina. ${ }^{3}$ The clinical consequences of pelvic organ prolapse (POP) are difficulty in voiding and evacuating stool, compromised sexual function, urinary and fecal incontinence, or a general feeling of discomfort due to a bulge in the vagina. The condition has been described as a "hidden epidemic" due to the large number of (76\%) of women experiencing POP symptoms at some stage of life, ${ }^{4,5}$ and it accounts for $20 \%$ of women awaiting gynecologic surgery in the UK. ${ }^{6}$ There is an estimated $19 \%$ lifetime risk of surgical requirement for POP. ${ }^{7}$ Primary factors that strongly influence the appearance of POP are childbearing, ${ }^{4,5,8}$ age, and high body mass index. ${ }^{4,5}$ Strong correlations between the incidence of POP and pregnancies, assisted deliveries including vacuum and particularly forceps, ${ }^{8}$ birth weight, the number of vaginal deliveries, ${ }^{9}$ and older age at first delivery have also been reported.,

A commonly used method for the evaluation of POP and integrity of the pelvic floor is the pelvic organ prolapse quantification (POP-Q) scoring system, developed as a six defined

*Address all correspondence to: Luke A. Parkinson, E-mail: luke.parkinson@ flinders.edu.au point anatomical measurement system with three additional general measurements to enhance both clinical and academic quantification of POP. The system was introduced to standardize terminology and measurement and uses the hymen as the point of reference to quantify the mobility and dimensions of specific internal and external anatomic locations during a pelvic examination. ${ }^{10,11}$ POP-Q evaluation provides an objective sitespecific system approach to communicate POP severity between practitioners, but it fails to identify the underlying cause of the problem and lacks the ability to provide data that can be used to develop understanding of the mechanism of POP. The system is arguably difficult to learn and overall adoption is not universal.

The need to measure and quantify the strength and structural integrity of the vaginal wall and its surrounding structures is exemplified by the number of investigators performing measurements of intravaginal pressure, ${ }^{12,13}$ vaginal/levator ani closure force ${ }^{14}$ and developing devices to measure vaginal tissue mechanical properties. ${ }^{15,16}$ Constantinou et al. ${ }^{17}$ developed a probe for making such measurements whereby two leaf-spring cantilevers were attached to a rod and inserted in the vagina. As the rod was withdrawn from the vagina, diametrically opposed cantilevers containing force transducers generated deflection measurements to describe the mechanical properties of the anterior and posterior vaginal walls. This technique measured vaginal closure force during voluntary and cough-induced pelvic floor muscle contractions but only addressed two points of the vagina at a time. Guaderrama et al. ${ }^{18}$ used a water perfused

$1083-3668 / 2016 / \$ 25.00$ @ 2016 SPIE 
catheter and a rapid pullback method to determine the vaginal pressure profile at rest and during levator ani contraction, determining the variable pressures present along the walls of the vagina. A single-point vaginal pressure measurement device developed by Arora et al. ${ }^{12}$ uses a wireless device for the measurement of intravaginal pressure during physical activity. A speculum device with rigid, dilatable blades, developed by Ashton-Miller et al., ${ }^{14}$ uses a strain gauge on each blade in an effort to determine contraction force of the levator ani muscle, a key support structure maintaining the integrity of the pelvic floor. Like many of the devices described to date, this instrument suffered from an inability to discern the difference between the contraction force of the physical structure (levator ani) from the force imparted on the device by intra-abdominal pressure (IAP), which fluctuates with tidal breathing and varies dramatically during events such as a cough, Valsalva, or voluntary pelvic floor activation. This device has been revised to separate IAP from levator ani contractile force and the reaction force of the symphysis pubis, but pressure distribution through the whole vagina is not measured. ${ }^{19}$

Magnetic resonance imaging (MRI) has been used effectively for several dynamic studies. ${ }^{20,21}$ However, access to MRI scanners is expensive and limited, particularly in remote areas. Furthermore, MRI images take over a second to acquire. ${ }^{21}$ MRI does not yet provide the temporal resolution to investigate the most rapid, dynamic events such as a cough or sneeze.

While the above techniques all provide invaluable information on the integrity of the vaginal wall, there is a need for a rapid, accurate, and quantifiable test that can provide distributed dynamic pressure measurement along the full vaginal length. Such measurements would accurately determine the precise location of the damaged vaginal tissue in both symptomatic and preclinical POP.

Optical fiber sensors have become increasingly attractive in biomedical sensing since they are flexible, can be multiplexed to create multiple discrete sensing locations, and are electromagnetically inert. The advent of fiber-Bragg gratings (FBGs) has enabled the development of distributed pressure, temperature, and strain measurements. FBGs are localized sensors inscribed into the core of an optical fiber that are extremely sensitive to strain due to its effect on the optical path length through the grating. The characteristic (unstrained) Bragg wavelength $\left(\lambda_{\mathrm{B}}\right)$ of FBGs written at discrete points along a fiber may be varied, allowing gratings to be wavelength encoded, which in turn permits distributed sensing using wavelength division multiplexing techniques. ${ }^{22}$

One complication that often confounds the use of FBGs as strain gauges is their pronounced sensitivity to changes in temperature. Wang et al. ${ }^{23}$ introduced an a thermal FBG pressure sensor design that effectively removed thermal artefact by using a pair of FBG-inscribed fibers wound together in a double helix, so the thermal effects were substantially removed. This technique has been subsequently employed to make distributed pressure measurements under bandages used in compression therapy. ${ }^{24}$

We have adapted this FBG-based distributed measurement technique to make an instrumented vaginal speculum with sensors located every $10 \mathrm{~mm}$ along both the upper and lower blades. This allows distributed pressure measurements to be made in the vagina to identify changes in support and possible defects in pelvic supporting tissue created by pregnancy and childbirth. The device has been validated in an ovine model comprising nulliparous and multiparous ewes and compared in parallel with a modified clinical POP-Q assessment to identify vaginal wall weakness (similar to POP-Q in women).

\section{Methods}

A standard stainless steel duckbill bivalve speculum was modified so that the upper and lower blades remained parallel during dilation. The speculum could be locked at any dilation between 0 and $20 \mathrm{~mm}$. At zero dilation, the separation of the upper and lower sensor regions is $\sim 15 \mathrm{~mm}$.

\subsection{Sensor Arrays}

Fiber-optic pressure sensing arrays with seven sensing elements spaced $10 \mathrm{~mm}$ apart were fabricated as described by Wang et al. $^{23}$ (Fig. 1) and bonded to both the upper (seven sensors) and lower blades (seven sensors) of the modified speculum (Fig. 2).
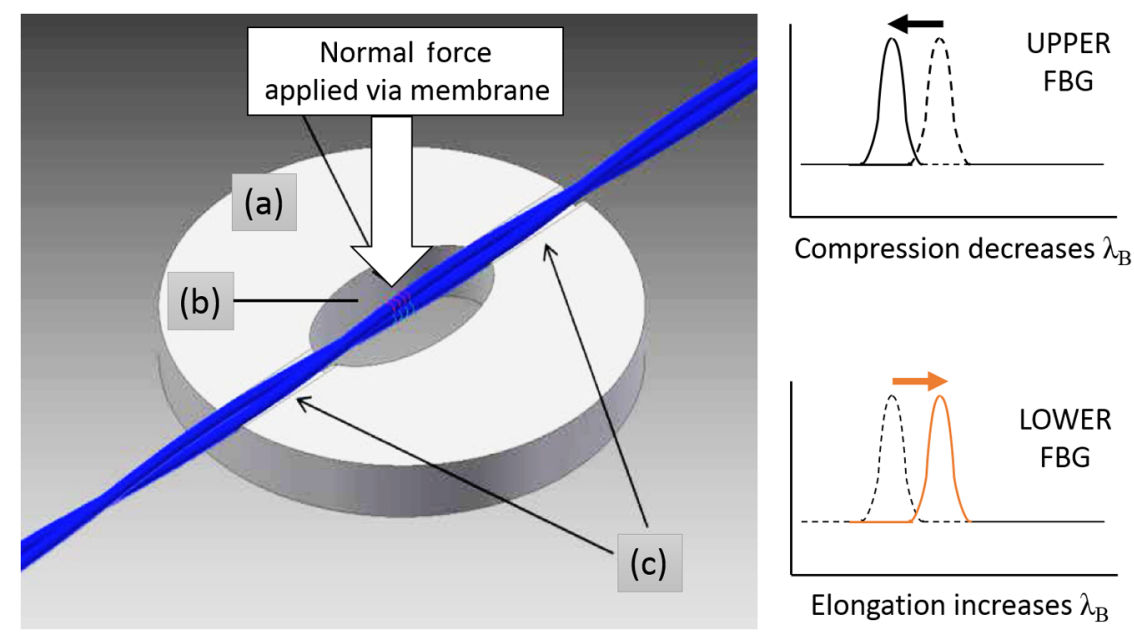

Fig. 1 A schematic adapted with permission from Ref. 23 showing the helically paired-FBG orientation. Each sensor is comprised of (a) metallic annular support, (b) FBG pair oriented above and below each other, and (c) glue bonds. A force applied normally to the plane of the solid substrate causes compression of the top fiber and elongation of the bottom fiber. 


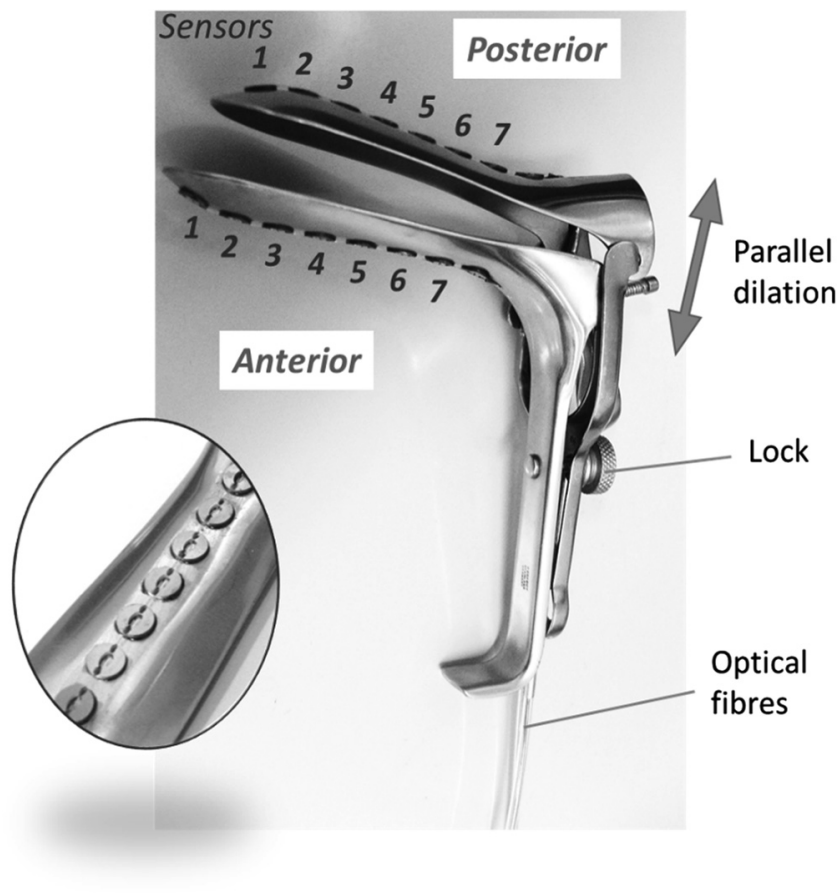

Fig. 2 Modified speculum showing sensors arranged along upper (posterior) and lower (anterior) blades (detail inset).

In brief, draw tower grating (DTG) optical fibers with FBGs were written at $10 \mathrm{~mm}$ spacing along their length with $2 \mathrm{~nm}$ spectral separation between each grating (FBGS Technologies $\mathrm{GmbH}$, Germany). Fiber pairs were then wound together in a double helix pattern with a pitch equal to the spatial separation of the grating pairs. The fibers were then bonded to an annular metallic substrate on each side of the FBG pair with the FBGs positioned above and below each other in the plane normal to the substrate (see Fig. 1). Deflection of the fibers due to a force applied normal to the substrate causes relative compression in the top fiber (reducing the Bragg wavelength of the FBG) compared with elongation in the bottom fiber (increasing the Bragg wavelength). The applied force is, therefore, proportional to the differential change in wavelength of the FBG pair. Changes in temperature affect both FBGs equally, making the force measurement independent of temperature. Each blade of the speculum was covered with a separate latex sleeve to provide a membrane protect the fibers and to deflect the underlying FBG in response to applied force. The fiber arrays were connected to a four-channel optical interrogator (FBGscan 804D, FBGS, Belgium) allowing the Bragg wavelengths of all FBGs to be monitored in real time.

The device was calibrated by applying a known pressure to each sensor using a pneumatically inflated bladder. In practice, this type of sensor measures a contact force that relates to the compliance of the vaginal wall. However, since the obstetrics and gynecology community is more familiar with pressure measurement, the sensors arrays were calibrated in pressure units of $\mathrm{mmHg}$ applied via the pneumatic cuff and $\mathrm{mmHg}$ were used for all subsequent analyses. Figure 3 shows the sensitivity of each individual sensor as a change in Bragg wavelength per unit of applied pressure.

The temperature insensitivity of the double-helix sensor configuration was verified by applying calibrated loads to the sensors as the temperature of the speculum was varied between

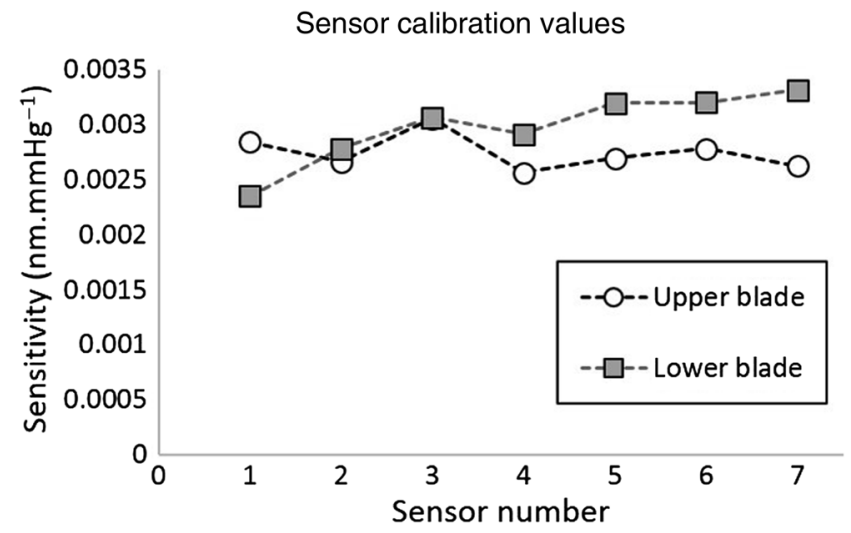

Fig. 3 Change in Bragg wavelength per $\mathrm{mmHg}$ applied pressure for each sensor on upper and lower speculum blades.

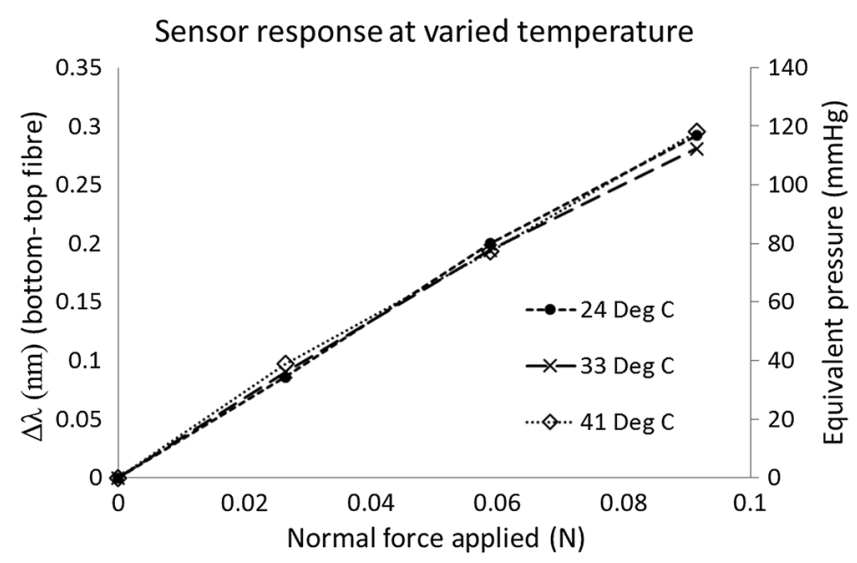

Fig. 4 The difference in wavelength between top and bottom fibers in response to the application of calibrated loads at three temperatures. Differences in Bragg wavelengths are shown on the primary axis. Equivalent pressures for a typical calibration value of $0.0025 \mathrm{~nm} \mathrm{mmHg}^{-1}$ are shown on the secondary axis for reference.

$24^{\circ} \mathrm{C}$ and $41^{\circ} \mathrm{C}$. Figure 4 shows the response of a typical sensor at three different temperatures. The measured force inferred from the differential change in Bragg wavelength remained unchanged as the temperature increased.

\subsection{Distributed Pressure Measurements in Ovine Vagina}

The experimental procedures were approved by the Monash Medical Centre Animal Ethics Committee A (MMCA/2013/ 38). Sheep were housed in paddocks of the Monash University Animal Service facilities in compliance with the National Health and Medical Research guidelines for the care and use of laboratory animals. This study examined 15 Border Leicester Merino ewes of which 3 were nulliparous (age 2 years) and 15 were parous, having delivered between 1 and 7 lambs in 1 to 4 pregnancies. The parous ewes ranged in age from 3 to 5 years, and the last lambs were born 6 months prior to this study.

Conscious sheep were constrained in a standard commercial $\mathrm{v}$-drive, and a modified POP-Q assessment was performed on each conscious animal by experienced gynecologists (A.R., N.Y.). The measurements were modified to suit the ovine pelvic anatomy as shown in Fig. 5. In these measurements, tissue laxity 


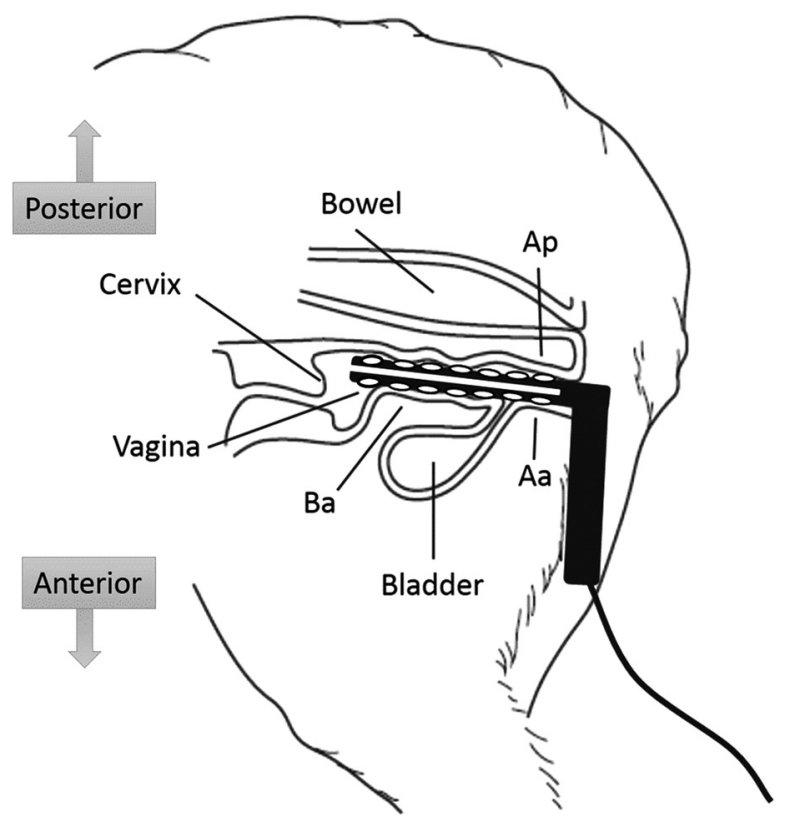

Fig. 5 Positioning of the fiber-optic speculum in relation to the ovine pelvic anatomy and the locations of the associated sites for POP-Q measurements ( $\mathrm{Aa}, \mathrm{Ba}, \mathrm{Ap})$. The upper speculum blade is positioned along the posterior vaginal wall incorporating $\mathrm{Ap}$ (distal posterior), with the lower blade positioned along the anterior wall incorporating $\mathrm{Ba}$ (proximal anterior vagina) and Aa (distal anterior vagina) regions.

was determined by using manual traction with forceps on the tissue in three regions of the vagina ( $\mathrm{Aa}, \mathrm{Ba}, \mathrm{Ap}$ ) (Fig. 3), using the urethra as a reference point for $\mathrm{Ba}$ (proximal anterior) and the point at which the inner mucous membrane of the vagina joins the skin in the distal vagina for Aa (distal anterior) and Ap (distal posterior). Immediately after vaginal wall displacement measurements were taken, the fiber-optic speculum was used to measure the force exerted by the vaginal walls. The speculum was inserted until the hilt of the blades contacted the perineal body in an orientation such that the upper and lower blades were in contact with the posterior and anterior vaginal wall, respectively (Fig. 5). Since pressure increased on each of the sensors on insertion, any sensors that remained exposed outside the vagina were readily identified.

Pressure was recorded at $10 \mathrm{~Hz}$ using custom software developed in LabVIEW (National Instruments, Texas). Pressure profiles along both anterior (lower blade) and posterior (upper blade) vaginal walls were recorded for the undilated speculum after which the blades were dilated at 5-mm increments up to a maximum of $20 \mathrm{~mm}$ unless significant discomfort was observed. The speculum blades were then returned to the undilated state in the same 5-mm increments (relaxation). The pressure profiles were recorded as static measurements at each stage during both dilation and relaxation to allow direct comparison between subjects. Events such as struggling, urination, sneezing, stamping gestures, and defecation were noted, and measurements were taken when the ewe was as passive as possible.

\subsection{Statistical Analysis}

Data are presented as mean \pm standard error of the mean (SEM). The cohort was divided into groups of nulliparous and parous ewes, and the overall pressure as well as the discrete pressure on each individual sensor was compared by Student's $t$-tests. $P<0.05$ was considered statistically significant.

\section{Results}

The 10-Hz data acquisition was adequate to obtain pseudocontinuous measurements of pressure fluctuations from all sensor locations. Measured pressures ranged from 0 to $300 \mathrm{mmHg}$. Data were recorded when the ewes were in a passive state, and dynamic variations associated with activity of the animals within the V-drive, which were clearly visible in the pressure recordings, were ignored.

Figure 6 shows the mean (of anterior and posterior combined) pressure at $10 \mathrm{~mm}$ dilation recorded during the dilation and relaxation cycles for the seven sensor locations along the vagina, with sensor 1 located toward the cervix. Since it was not possible to reach the full $20 \mathrm{~mm}$ dilation in all animals, data recorded at $10 \mathrm{~mm}$ dilation were used for the analysis. In general, recorded pressures were higher during increasing dilation compared to the same measurement taken on relaxation, which was assumed to be due to the animal acclimatizing to the device. For nulliparous sheep, the overall pressure at $10-\mathrm{mm}$ separation during dilation was $17.5 \%$ higher than at $10-\mathrm{mm}$ separation during relaxation $(P<0.01$ by sensor-paired Student's $t$-test) (Fig. 6). For the parous group, the mean pressure was $38 \%$ higher in the preversus postmaximal dilation measurements $(P<0.01)$. It was decided to use the measurements taken during relaxation of the speculum as these appeared less affected by movement of the animal. The mean pressure across all sensors was $102 \pm 46 \mathrm{mmHg}$ for nulliparous ewes compared with a mean pressure of $39 \pm 23 \mathrm{mmHg}$ for parous ewes $(P<0.05)$. The difference between nulliparous and multiparous pressure readings was largest in the proximal vagina region, closest to the cervix, between sensors 1 and 3, with another point of higher pressure measured at sensor 5 .

Analysis of the pressure along each blade of the speculum identified some significant differences in the distribution of pressure between nulliparous and parous ewes (Fig. 7). Pressures were higher on the upper blade [posterior wall, Fig. 7(a)] for nulliparous ewes at each end for sensors 1 to 2 and for sensor $5(P<0.01)$. The pressure distribution on the lower blade showed no difference between groups in the distal vagina. Much higher pressures were measured for the anterior

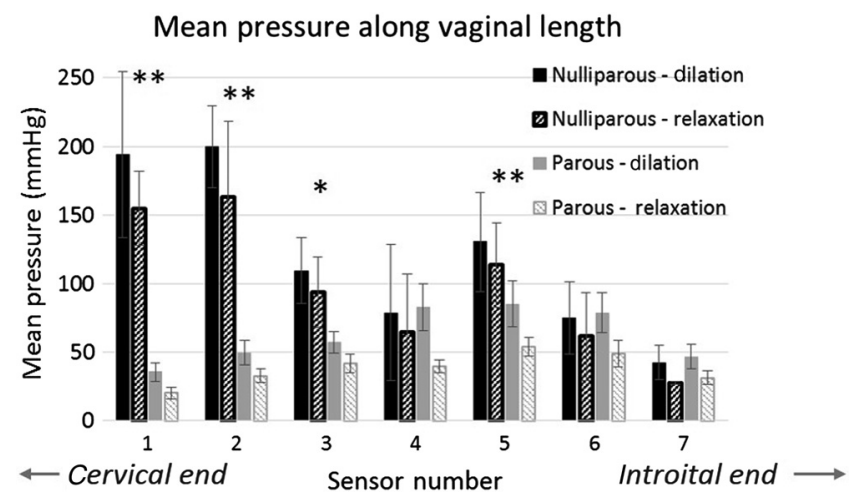

Fig. 6 Mean pressures \pm SEM for sensor locations 1 to 7 upon initial dilation to $10 \mathrm{~mm}$ and at $10-\mathrm{mm}$ dilation after maximal stretch $(20 \mathrm{~mm})$. A difference in pressure distribution for nulliparous versus parous sheep in absolute values before and after maximal dilation was observed. Differences in pressure between nulliparous and parous ewes are indicated $\left({ }^{\star *} P<0.001 ;{ }^{\star} P<0.05\right)$. 

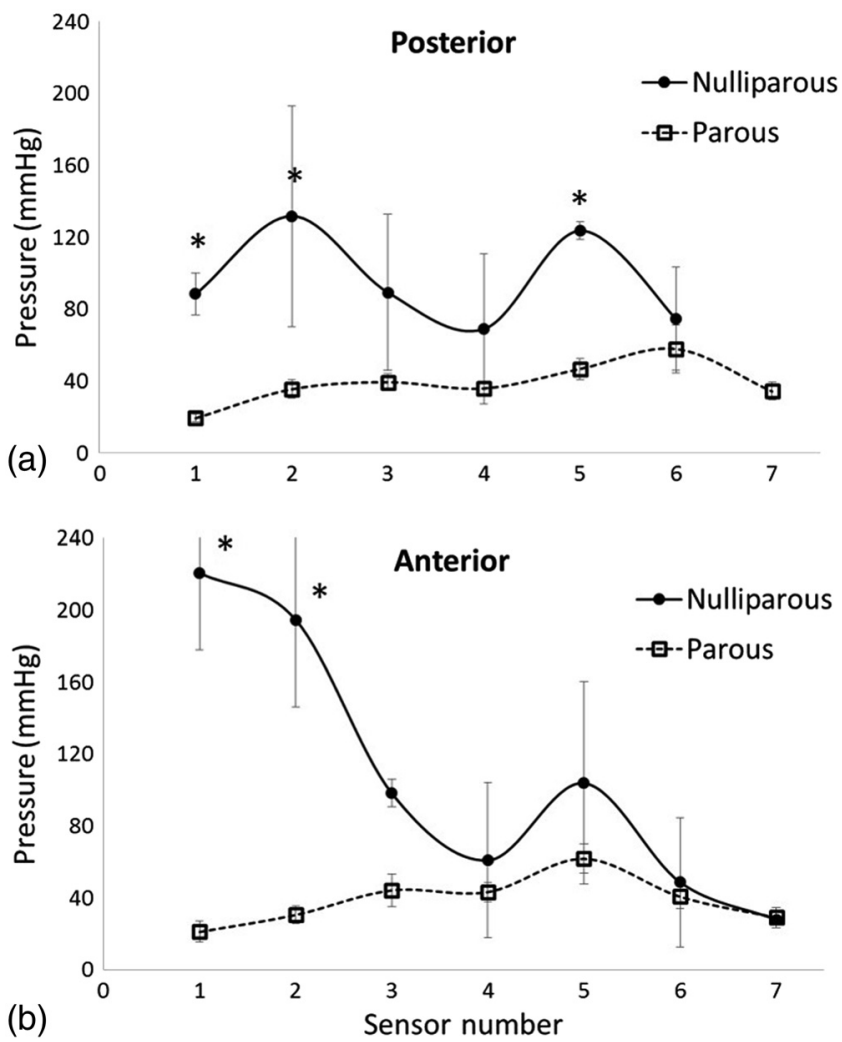

Fig. 7 Distribution of pressure on (a) posterior and (b) anterior blades at $10 \mathrm{~mm}$ dilation for nulliparous $(n=3)$ and parous $(n=12)$ ewes. Data are mean \pm SEM ${ }^{*} P<0.01$ (nulliparous versus parous).

wall of the proximal vagina in the nulliparous group compared with those measured for the parous group. For sensors 1 and 2 in the proximal anterior vagina, the pressures were significantly different $(200 \pm 60 \mathrm{mmHg}$ for the nulliparous ewes compared with $40 \pm 20 \mathrm{mmHg}$ for the parous group, $P<0.001)$ [Fig. 7(b)]. Poor sensor contact at the introitus prevented reliable measurements from being recorded at sensor 7 on the posterior blade for nulliparous ewes.

Within the group of parous ewes, POP-Q measurements identified a subgroup where excessive displacement was observed in the $\mathrm{Ba}$ (proximal anterior vagina) position due to increased tissue laxity. To test whether this vaginal wall laxity is detected as a change in pressure measurement for the respective sensors on the speculum, the parous ewe pressure data were divided into two groups based on displacement values in the $\mathrm{Ba}$ region of the proximal anterior vagina ( -3 no displacement versus values $>-2$ for $\mathrm{Ba}$ displacement group). Figure 8 shows the mean pressure distribution on the anterior vaginal wall for parous ewes only. For those ewes with Ba displacement, the region from sensors 1 to 3 , which corresponds to the $\mathrm{Ba}$ region, showed lower mean pressure $(22.8 \pm 4.4 \mathrm{mmHg})$ than the same sensors in those ewes without $\mathrm{Ba}$ displacement $(44 \pm 12 \mathrm{mmHg}), P<0.05$. Conversely in the distal vagina region (sensors 5 to 7 ), the group that exhibited $\mathrm{Ba}$ displacement showed higher pressure $(57 \pm 8 \mathrm{mmHg})$ than the group that did not show $\mathrm{Ba}$ displacement $(32 \pm 11 \mathrm{mmHg})$.

\section{Discussion}

A modified optical fiber-speculum has been successfully used to discern general variations in pressure applied by the vaginal
$10 \mathrm{~mm}$ dilation Anterior wall Parous Ba displacement vs No Ba displacement

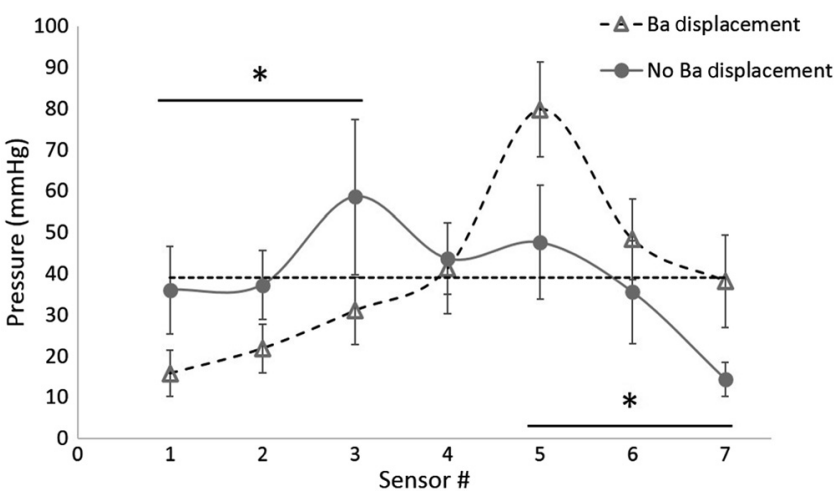

Fig. 8 Pressure distribution (mean \pm SEM) along the anterior vaginal wall for parous ewes exhibiting vaginal displacement at the Ba position (> -2 ) as measured in the modified POP-Q assessment (dotted line) and parous ewes with no displacement at the $\mathrm{Ba}$ location $(-3)$ (solid line). The mean pressure across all sensors for each of these groups was nominally equal at $39 \mathrm{mmHg}$, indicated by the horizontal dashed line to highlight the variation in the relative distribution of pressure for the two groups. The horizontal bars and asterisk highlight pressure regions at sensors 1 to 3 and 5 to 7 where pressure differs significantly between the two groups of ewes (Ba displaced, $n=5$; no Ba displacement, $n=5$; ${ }^{*} P<0.05$ ).

wall, as well as resolving the spatial variation in this pressure. A key finding in this first assessment of our device in the ovine model is that the distribution of pressure, as well as the mean pressure, is significantly different between nulliparous and parous ewes. Our data demonstrate lower support in parous ewes, suggesting a weakening of the vaginal wall that may be derived from injury associated with birth. ${ }^{25}$ Furthermore, the pressure sensor device discriminated between parous ewes with normal and abnormal vaginal displacement measurements based on modified POP-Q measurements. The identification of a region (equivalent to $\mathrm{Ba}$ of the POP-Q in humans) of less support in the proximal region of the anterior vaginal wall of parous ewes may be linked to irreversible traumatic changes associated with pregnancy and parturition. Guaderrama et al. ${ }^{18}$ provide some insight into the variability of pressure distribution along the length of the human vagina as well as around its circumference by making dynamic measurements from a quadrant water-perfused catheter. As the catheter was drawn along the length of the vagina, it showed a distinct region of high pressure through the mid to distal vagina of around 35 to $40 \mathrm{mmHg}$ in nulliparous women. Their findings highlight the need for distributed pressure measurement but cannot be directly compared with ours due to the obvious differences in size of the instruments and pelvic anatomy. We have not found any similar data in the published literature that allow us to relate our distributed pressure results to others in the ovine model.

We have observed that the use of rigid, movable blades enabled the distribution of vaginal wall pressure to be measured. While the mean pressure across all sensors on the anterior wall is nominally the same for parous ewes both with and without vaginal tissue displacement in the $\mathrm{Ba}$ region, we observed differences in the distribution of this pressure at the specific sensor sites that correlated with the location of the Ba measurements that differed between these two groups (Fig. 8).

This colocation of differences in the modified POP-Q and pressure profile measurements suggests that the instrumented 
speculum may provide an alternative means of identifying and localizing prolapse or provide a viable means of screening for elevated risk of prolapse in parous women.

\section{Limitations of the Study}

Our ability to draw physiological conclusions based on these data is limited by the small number of ewes available with known birth histories for the investigation and the large variance of the data. Contributing factors to the large variance in our measurements, in addition to the standard physiological variations, were the posture of the sheep in the v-drive and their level of agitation and activity. Discomfort of the ewes and nervous clenching on the speculum may also have confounded the data. Integration of pressure readings over a slightly longer period or repeated dilation and relaxation of the speculum during measurement may reduce the artefact attributable to animal movement.

Relation of these data recorded in this ovine model to human physiology is limited due to the clear differences between human and ovine anatomy and orientation (as reflected by the modifications made to the POP-Q examination to suit the ovine anatomy). Sheep are prostrate quadrupeds with gravity acting on the pelvic structure in an anterior direction, which will clearly have a large impact on results despite the closely matched vaginal dimensions of the two species. For example, the weight of the bladder on a poorly supported anterior vaginal wall causes prolapse of the bladder into the vagina (cystocoele) in humans, ${ }^{19}$ whereas in the ovine model gravity draws the bladder away from the proximal anterior vagina. However, these preliminary results clearly demonstrate the utility of the fiber-optic speculum as a device for detecting variations in vaginal pressure along the entire vaginal length on both anterior and posterior walls.

\section{Conclusions}

These preliminary data verified the ability for an instrumented speculum containing arrays of sensors along the upper and lower blades to measure variations in pressure along the length of the ovine vagina and demonstrated its ability to discern differences in the overall magnitude and distribution of pressure due to physiological variation. We have verified the ability of the modified optical fiber-speculum device to discern differences in vaginal pressure in the ovine model. Our measurements demonstrated a general decrease in the elasticity or support of the vagina with parity, and there was some agreement between a relevant modified POP-Q measurement and the speculum data. Further work is required to more vigorously validate the utility of our speculum device and to gain a better understanding of how the information it provides relating to the distribution of support in the vagina should be interpreted and related to the appearance of POP.

In summary, we have demonstrated a high-resolution vaginal pressure measurement device that will provide quantified, spatially resolved, and dynamic pelvic support information that is likely to be of great benefit in dynamic assessment as it is developed for use in the conscious human patient where dynamic stimuli can be requested.

\section{Disclosures}

None of the authors has a financial interest or conflict of interest in this work.

\section{Acknowledgments}

This work was supported by the National Health and Medical Research Council (NHMRC) of Australia Project Grant No. 1081944 (C.E.G., J.A.W., J.A., A.R.), a NHMRC Senior Research Fellowship No. 1042298 (C.E.G.,), and the Victorian Government's Operational Infrastructure Support Program. J.A. was funded by a South Australian Premier's Professorial Fellowship.

\section{References}

1. J. A. Ashton-Miller and J. O. DeLancey, "On the biomechanics of vaginal birth and common sequelae," Апnи. Rev. Biomed. Eng. 11, 163-176 (2009).

2. J. O. L. DeLancey, "The hidden epidemic of pelvic floor dysfunction: achievable goals for improved prevention and treatment," Am. J. Obstet. Gynecol. 192(5), 1488-1495 (2005).

3. M. S. Baggish and M. M. Karam, Atlas of Pelvic Anatomy and Gynecologic Surgery, 3rd ed., Elsevier Health St. Louis (2006).

4. J. E. Jelovsek, C. Maher, and M. D. Barber, "Pelvic organ prolapse," Lancet 369, 1027-1038 (2007).

5. S. Swift et al., "Pelvic organ support study (POSST): the distribution, clinical definition, and epidemiologic condition of pelvic organ support defects," Am. J. Obstet. Gynecol. 192, 795-806 (2005).

6. L. Cordozo, "Prolapse," in Dewhurse's Textbook of Obstetrics and Gynaecology for Postgraduates, C. R. Whitfield, Ed., pp. 642-652, Blackwell Science, Oxford (1995).

7. F. J. Smith et al., "Lifetime risk of undergoing surgery for pelvic organ prolapse," Obstet. Gynecol. 116(5), 1096-1100 (2010).

8. H. P. Dietz and M. J. Bennett, "The effect of childbirth on pelvic organ mobility," Obstet. Gynecol. 102(2), 223-228 (2003).

9. M. Gyhagen et al., "Prevalence and risk factors for pelvic organ prolapse 20 years after childbirth: anational cohort study in singleton primiparae after vaginal and caesarean delivery," Br. J. Obstet. Gynaecol. 120, 152-160 (2013).

10. D. R. Bland et al., "Use of the pelvic organ prolapse staging system of the international continence society, American urogynecologic society, and society of gynecologic surgeons in perimenopausal women," Am. J. Obstet. Gynecol. 181, 1324-1328 (1999).

11. R. C. Bump et al., "The standardization of terminology of pelvic organ prolapse and pelvic floor dysfunction," Am. J. Obstet. Gynecol. 175, 10-17 (1996).

12. A. S. Arora et al., "Clinical evaluation of a high-fidelity wireless intravaginal pressure sensor," Int. Urogynecol. J. 26, 243-249 (2015).

13. J. A. Kruger et al., "Design and development of a novel intra-vaginal pressure sensor," Int. Urogynecol. J. 24, 1715-1721 (2013).

14. J. A. Ashton-Miller, J. O. L. DeLancey, and D. N. Warwick, "Method and apparatus for measuring properties of the pelvic floor muscles," U.S. Patent No. \# 6,468,232 B1 (2002).

15. V. Egorov, H. van Raalte, and V. Lucente, "Quantifying vaginal tissue elasticity under normal and prolapse conditions by tactile imaging," Int. Urogynecol. J. 23, 459-466 (2012).

16. V. Egorov, H. Van Raalte, and A. P. Sarvazyan, "Vaginal tactile imaging," IEEE Trans. Biomed. Eng. 57(7), 1736-1744 (2010).

17. C. E. Constantinou et al., "Evaluation of the dynamic responses of female pelvic floor using a novel vaginal probe," Ann. N. Y. Acad. Sci. 1101, 297-315 (2007).

18. N. M. Guaderrama et al., "The vaginal pressure profile," Neurol. Urodyn. 24, 243-247 (2005).

19. J. A. Ashton-Miller et al., "Validity and reliability of an instrumented speculum designed to minimize the effect of intra-abdominal pressure on the measurement of pelvic floor muscle strength," Clin. Biomech. 29, 1146-1150 (2014).

20. D. M. Spahlinger et al., "Relationship between intra-abdominal pressure and vaginal wall movements during Valsalva in women with and without pelvic organ prolapse: technique development and early observations," Int. Urogynecol. J. 25(7), 873-881 (2014).

21. A. Summers et al., "The relationship between anterior and apical compartment support," Am. J. Obstet. Gynecol. 194(5), 1438-1443 (2006). 
22. J. W. Arkwright et al., "In-vivo demonstration of a high resolution optical fiber manometry catheter for diagnosis of gastrointestinal motility disorders," Opt. Express 17(6), 4500-4508 (2009).

23. D. H.-C. Wang et al., "An optical fiber Bragg grating force sensor for monitoring sub-bandage pressure during compression therapy," Opt. Express 21(17), 19799-19807 (2013).

24. J. W. Arkwright, D. H.-C. Wang, and V. Patton, "An optical fibre tape sensor for monitoring sub-bandage pressures: progress towards an 'ideal sensor'," J. Lymphoedema 8(1), 24-28 (2013).

25. B. M. Couri et al., "Animal models of female pelvic organ prolapse: lessons learned," Expert Rev. Obstet. Gynecol. 7(3), 249-260 (2012).

Luke A. Parkinson is a research fellow at Flinders University. He has expertise in the physical, chemical, and life sciences as well as in mechanical design engineering. His publications and patents span mineral processing and microengineering to biosensing. He is now at The Medical Device Research Institute working on optical fiber technologies for medical diagnostics.

Caroline E. Gargett is a National Health and Medical Research Council senior research fellow, deputy director of the Ritchie Centre, and head of the Endometrial Stem Cell Biology Laboratory. She discovered adult stem cells in the highly regenerative endometrial lining of the uterus and is developing the endometrial mesenchymal stem cell for treating pelvic organ prolapse using tissue engineering approaches.

Natharnia Young is a certified urogynecologist at Monash Health, Victoria, and collaborates with the research team through Hudson Institute and Flinders University. Her main areas of interest are research in urinary incontinence and prolapse and basic science research in tissue engineering.

Anna Rosamilia is a certified urogynecologist who heads the pelvic floor unit at Monash Medical Centre. She is an examiner for MBBS O $\& G$ and $C U$. She is the current treasurer of the International
Urogynaecological Association, is a member of the Urogenital Prostheses Clinical Advisory Group and was on the board for the Australasian Endoscopy and Surgical Society. Her main areas of interest are clinical trials in urinary incontinence and basic research in tissue engineering and pelvic organ prolapse.

Aditya V. Vashi is an experimental scientist in the Manufacturing Division of The Commonwealth Scientific and Industrial Research Organization (CSIRO), Australia. He is a chemical engineer with a master's degree in biotechnology and has extensive research experience in the field of biomaterials and tissue engineering. He has a keen interest in cell-material interactions, 3-D cultures, and scaffold fabrication.

Jerome A. Werkmeister is a tissue engineer with expertise in materials and scaffold design, cell and matrix biology, and immunology. He serves on the editorial board of several international biomaterial journals, has published 186 papers, and holds several patents. He was a cofounder of the Australasian Society for Biomaterials and Tissue Engineering and has been internationally recognized for his scientific contributions to the field of biomaterials science by the award of fellow, biomaterials science and engineering.

Anthony W. Papageorgiou is a trade qualified machinist and mechanical engineer currently specializing in fiber-optic sensing technologies at the Medical Device Research Institute, Flinders University.

John W. Arkwright has an extensive background in optical fiber device technology. He has worked in both industrial R\&D and academic roles, always with an emphasis on translational technologies. $\mathrm{He}$ has experience at many different levels from fundamental research to production line engineering. He is currently at Flinders University working on fiber-optic sensors for in vivo diagnostics. He is an MD of Arkwright Technologies Pty. Ltd., an Adelaide-based company commercializing fiber-optic sensing technologies. 